\title{
On the Nondimensionalization Process in Complex Problems: Application to Natural Convection in Anisotropic Porous Media
}

\author{
I. Alhama, ${ }^{1}$ M. Cánovas, ${ }^{2}$ and F. Alhama ${ }^{3}$ \\ ${ }^{1}$ Civil Engineering Department, Technical University of Cartagena, 30203 Cartagena, Spain \\ ${ }^{2}$ Mining, Geologic and Cartographic Engineering Department, Technical University of Cartagena, 30203 Cartagena, Spain \\ ${ }^{3}$ Applied Physics Department, Technical University of Cartagena, Murcia, 30203 Cartagena, Spain
}

Correspondence should be addressed to M. Cánovas; manuel.canovas@upct.es

Received 11 February 2014; Accepted 18 March 2014; Published 22 June 2014

Academic Editor: Yongqi Wang

Copyright (C) 2014 I. Alhama et al. This is an open access article distributed under the Creative Commons Attribution License, which permits unrestricted use, distribution, and reproduction in any medium, provided the original work is properly cited.

\begin{abstract}
The nondimensionalization of the equations governing a given problem is a direct, relatively easy, and low-cost way to provide interesting information, the dimensionless groups that rule the problem and define its final patterns of solution. In complex problems, however, this technique frequently does not provide the precise and complete set of monomials we are looking for. The use of discrimination in the process of nondimensionalization, the detailed application of which is explained in this paper, always leads to a minimum set of parameters, which, separately, determine the solution of the problems. In addition, the physical meaning and order of magnitude of these discriminated monomials are also provided by the discrimination. The technique is applied to the coupled problem of natural convection between horizontal plates heated from below, containing an anisotropic porous medium.
\end{abstract}

\section{Introduction}

The search for the minimum group of parameters that rule the solution to a given problem, especially if it is complex and can only be solved numerically, is perhaps the main goal of experimental, numerical, and theoretical research and great effort has been put into the same. Apart from experimental work, there are basically two techniques that are used: the application of dimensional analysis and the nondimensionalization of the governing equations. The cost of these nonexperimental techniques is very low in light of the advantages they provide.

The first procedure [1-3], which is followed in many textbooks for academic purposes, starts from the correct set of relevant quantities whose potential flows balance each other in the conservation laws of the problem, as well as of physical characteristics that related the relevant quantities in the conservation laws. By choosing a suitable dimensional basis, formed by the fundamental quantities of the theory in which the problem is involved, and applying the pi theorem, it is possible to derive the final dimensionless groups. However, as mentioned above, this technique is scarcely used and few works that use it can be found in the scientific literature
[4-9]. Two fundamental reasons justify this absence and its progressive abandonment. Firstly, a deep understanding of the theory involved in the physical process is required for correct formulation of the complete and unique list of variables; the researcher frequently does not include in this list hidden quantities that are not mentioned in the statement of the problem but which, however, take part in the balances or conservation laws; this occurs, for example, in 2D scenarios in which one of the lengths extends beyond the limits of where the phenomenon is mainly located. Secondly, even when the list of relevant variables is correctly formulated, the classical application of pi theorem does not always provide the independent dimensionless groups unless discrimination is taken into account in the nondimensionalization procedure. The concept of discrimination as an extension of dimensional analysis is introduced by Madrid and Alhama [10] in the field of convective heat transfer and used by these and other authors for the derivation of the correct dimensionless groups [11-13].

The use of the more formal nondimensionalization technique, however, is extensively used both in advanced textbooks and in the scientific literature in general [14-17]. In its classical form, this technique does not distinguish 
between scalar and vectorial quantities but chooses any length, independently of its spatial direction, to make all the independent spatial variables dimensionless. The same occurs with velocities and other quantities of a vectorial character. This nondiscriminated (classical) procedure frequently leads to results that can be improved. Many of the dimensionless numbers, such as Reynolds, Rayleigh, Nusselt, and Grashof (or their reverse), have very high or very low values compared with unity and lack any sense in terms of quantity balances that counteract the physical process; indeed, this would make an interesting research subject. In contrast, the use of discrimination clearly improves the solution in fundamental aspects: the number of resulting groups is less, their order of magnitude is unity, and (as a consequence) they can be immediately interpreted physically as balances of quantities in the domains where they are applied. Madrid and Alhama [17] and Madrid and Alhama [18] applied the discriminated nondimensionalization to solve the problems of laminar forced convection along a vertical plate and natural convection along an isothermal vertical plate, providing very precise results.

In this paper, mathematically orientated from the point of view of equations management but without losing the physical understanding of the problem, discriminated nondimensionalization is applied to a coupled, nonlineal, very complex problem involving many physical parameters, in order to demonstrate not only the formal rules of application, but also some complementary and interesting aspects derived from this technique, such as the potential existence of hidden quantities necessary for the definition of dimensionless dependent or independent variables. Subsequently, when dimensionless groups have been derived, the meaning and even the order of magnitude of these quantities can be elucidated. The problem, studied by Holzbecher and Yusa [19] and Holzbecher [20], refers to the natural heat convection between large horizontal plates, heated from below, containing an anisotropic porous medium. In this kind of problem, the flow is organized in close structures regularly distributed, named cells (or Bénard's cells), along the horizontal coordinate.

After the discriminated dimensionless groups have been obtained, many scenarios in which these groups retain the same value but in which the physical characteristics or geometrical parameters are changed are solved numerically in order to demonstrate the validity of the solutions. By making use of the physical meaning of the resulting groups, the order of magnitude of the principal unknown of the problem can be obtained.

\section{Governing Equations and Boundary Conditions}

The scenario studied is shown in Figure 1. It refers to a fluid-flow and heat-transport process-a natural convection problem-occurring between horizontal plates subjected to a thermal gradient and impermeable to fluid. The scenario is $2 \mathrm{D}$ rectangular, and the porous medium assumes anisotropy for the hydraulic and thermal conductivities.

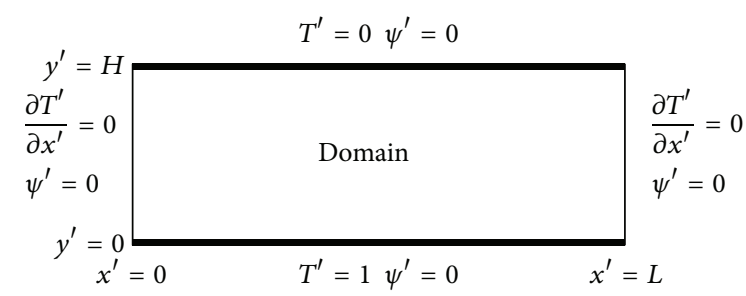

FIGURE 1: Natural convection between horizontal plates. Scheme of the problem.

2.1. Dimensional Form. In the steady state, the set of governing equations is formed by (i) the expressions of the horizontal and vertical components of the flow velocity, $u$ and $v$, respectively, derived from Darcy's law, (ii) the mass conservation equation, and (iii) energy conservation equation. For anisotropic $2 \mathrm{D}$ media, using rectangular coordinates, these are

$$
\begin{gathered}
u=-\left(\frac{K_{x}}{\mu}\right)\left(\frac{\partial P}{\partial x}\right), \quad v=-\left(\frac{K_{y}}{\mu}\right)\left\{\left(\frac{\partial P}{\partial y}\right)+\rho g\right\}, \\
\left(\frac{\partial u}{\partial x}\right)+\left(\frac{\partial v}{\partial y}\right)=0 \\
\left(\rho_{f} c_{p, f}\right) u\left(\frac{\partial T}{\partial x}\right)+\left(\rho_{f} c_{p, f}\right) v\left(\frac{\partial T}{\partial y}\right) \\
=k_{m, x}\left(\frac{\partial^{2} T}{\partial x^{2}}\right)+k_{m, y}\left(\frac{\partial^{2} T}{\partial y^{2}}\right) .
\end{gathered}
$$

In these equations $K$ is the hydraulic conductivity $(\mathrm{m} / \mathrm{s})$, $P$ the pressure $\left(\mathrm{N} / \mathrm{m}^{2}\right), \rho$ the fluid density $\left(\mathrm{kg} / \mathrm{m}^{3}\right), g$ the gravitational acceleration $\left(\mathrm{m} / \mathrm{s}^{2}\right), \mu$ the fluid viscosity $\left(\mathrm{kg} \mathrm{m}^{-1} \mathrm{~s}^{-1}\right), c_{p, f}$ the specific heat $\left(\mathrm{J} / \mathrm{Kg}^{\circ} \mathrm{C}\right), k$ the thermal conductivity $\left(\mathrm{W} / \mathrm{m}^{\circ} \mathrm{C}\right)$, and $T$ the temperature $\left({ }^{\circ} \mathrm{C}\right)$; finally, $x(m)$ and $y(m)$ are the independent variables. Subscript $m$ means average value, $f$ and $s$ refer to fluid and solid matrix, respectively, and $x$ and $y$ denote the anisotropic character of the parameter. Average thermal conductivity is defined in terms of the porosity, $\phi$ (dimensionless), and the fluid and solid matrix conductivities $\left(k_{f}\right.$ and $k_{s}$, resp.), $k_{m}=\left[\phi k_{f}+\right.$ $\left.(1-\phi) k_{s}\right]$.

Eliminating the pressure in the Darcy equation and making use of the Boussinesq approximation (a hypothesis widely used in most processes in this field, Holzbecher [20]),

$$
\rho=\rho_{0}-\rho_{0} \beta \Delta T=\rho_{0}(1-\beta \Delta T), \quad \text { or } \Delta \rho=-\rho_{0} \beta(\Delta T),
$$

where $\beta$ is the thermal expansion coefficient $\left({ }^{\circ} \mathrm{C}^{-1}\right)$, subscript 0 refers to a reference temperature, and the momentum equations are combined in the form

$$
\left(\frac{K_{y}}{K_{x}}\right)\left(\frac{\partial u}{\partial y}\right)-\left(\frac{\partial v}{\partial x}\right)=-\left(\frac{\rho K_{y} g \beta}{\mu}\right)\left(\frac{\partial T}{\partial x}\right) .
$$

This reduces the mathematical models to (2), (3), and (5). As regards the pressure variable, (1) would be used for deriving 
its changes from the characteristic velocities obtained in this work. To complete the problem, the following boundary conditions are assumed: temperature at the top and bottom boundaries is isothermal $\left(T_{b}>T_{t}\right)$, with $\Delta T=T_{b}-T_{t}$, while both left and right sides are adiabatic; in addition, all sides are impermeable to water flow:

$$
\begin{gathered}
T_{(x, y=0)}=T_{b}, \quad T_{(x, y=H)}=T_{t}, \\
\left(\frac{\partial T}{\partial x}\right)_{\text {left }}=\left(\frac{\partial T}{\partial x}\right)_{\text {right }}=0, \\
q_{(x, y=0)}=q_{(x, y=H)} q_{(x=0, y)} q_{(x=L, y)}=0,
\end{gathered}
$$

where $q$ is the fluid flow $(\mathrm{m} / \mathrm{s})$ and $L$ and $H$ define the geometry of the rectangular domain. The aspect ratio of the problem $L / H$ is sufficiently large, $L \gg H$.

\subsection{Discriminated Dimensionless Form}

2.2.1. The Reference Quantities. Discrimination forces the choice of different characteristic values, according to their spatial direction, to make the variables of vectorial character, velocity $(u$ and $v)$, and coordinates ( $x$ and $y$ ) dimensionless. On the one hand, as regards the characteristic lengths, although two discriminated lengths appear in the statement of the problem, one of them $(L)$ is not relevant since its value is large enough to neither influence the problem nor rule the solution. It is easy to deduce that the steady state patterns cannot be dependent on $L$ when $L$ is large enough; that is, patterns may cover the totality of the domain or a part of it, but its aspect remains invariable as $L$ increases. As a consequence, $L$ cannot be chosen as a horizontal reference quantity, and, since no more references exist, we assume a "hidden" length $\left(l_{x}^{*}\right)$ for this propose, an unknown whose physical meaning and value will be investigated later, after derivation of the dimensionless groups. In short, the reference quantities to make $x$ and $y$ dimensionless are $\left(l_{x}^{*}\right)$ and $H$, respectively. On the other hand, as regards velocities, the vertical reference can be derived directly from the first addend of the left part of (1) making $\Delta \rho=\Delta \rho_{\max }$, since the set of parameters " $\left(K_{y} / \mu\right)\left(\Delta \rho_{\max }\right) g$ " provides a characteristic value related to the vertical coordinate. Finally, we assume a new hidden quantity, $u_{x}^{*}$, as the horizontal reference velocity since this cannot be established directly or indirectly by the set of parameters contained in the statement. The length and velocity references are as follows:

for the $x$ coordinate: $l_{x}^{*}$,

for the $y$ coordinate: $l_{y}^{*}=H$,

for the vertical velocity, $v: v^{*}=-\left(K_{y} / \mu\right)(\Delta \rho) g=$ $-\left(K_{y} / \mu\right) \rho_{0} g \beta(\Delta T)$,

for the horizontal velocity, $u: u^{*}$,

for the temperature, $T: \Delta T=T_{b}-T_{t}$.
2.2.2. The Dimensionless Variables. Based on the above references, the following dimensionless variables can be defined:

$$
\begin{gathered}
x^{\prime}=\frac{x}{l_{x}^{*}}, \\
y^{\prime}=\frac{y}{H}, \\
u^{\prime}=\frac{u}{u^{*}}, \\
v^{\prime}=\frac{v}{v^{*}}=\frac{v \mu}{\left[\rho_{0} g \beta(\Delta T) K\right]}, \\
T^{\prime}=\frac{T}{\Delta T} .
\end{gathered}
$$

We assume that the dimensionless variables $x^{\prime}$ and $u^{\prime}$ range in the interval $[0,1]$, even though the references used in their definition are unknowns; this is a hypothesis that will be tested later. Furthermore, it is clear that $y^{\prime}$ and $v^{\prime}$ also range in the same interval. This property of the dimensionless variables will be used later in the dimensionless governing equation to assign a suitable order of magnitude to the discriminated groups we are looking for and to give a physical meaning and an order of magnitude to the "hidden" references.

2.2.3. The Dimensionless Equations. Firstly, we study the mass conservation equation because of its simplicity. Substituting (7)-(10) into (2) yields

$$
\left(\frac{u^{*} \partial u^{\prime}}{l_{x}^{*} \partial x^{\prime}}\right)+\left(\frac{v^{*} \partial v^{\prime}}{H \partial y^{\prime}}\right)=0
$$

or

$$
\left(\frac{u^{*} \partial u^{\prime}}{l_{x}^{*} \partial x^{\prime}}\right)+\left(\frac{\left[\rho_{0} g \beta(\Delta T) K_{y}\right] \partial v^{\prime}}{\mu H \partial y^{\prime}}\right)=0 .
$$

Reorganizing this equation by grouping all the parameters in the same addend, we can write

$$
\left(\frac{\mu H u^{*}}{\left[\rho_{0} g \beta(\Delta T) K_{y}\right] l_{x}^{*}}\right)\left(\frac{\partial u^{\prime}}{\partial x^{\prime}}\right)+\left(\frac{\partial v^{\prime}}{\partial y^{\prime}}\right)=0 .
$$

Note that the expressions within the parentheses are dimensionless.

Secondly, we will work with the momentum equation (5). Again, using the dimensionless variables, this equation can be written in the form

$$
\left(\frac{K_{y}}{K_{x}}\right)\left(\frac{u^{*} \partial u^{\prime}}{H \partial y^{\prime}}\right)-\left(\frac{v^{*} \partial v^{\prime}}{l_{x}^{*} \partial x^{\prime}}\right)=-\left(\frac{\rho K_{y} g \beta}{\mu}\right)\left(\frac{(\Delta T) \partial T^{\prime}}{l_{x}^{*} \partial x^{\prime}}\right)
$$

or, making use of (12) and simplifying in the dimensionless form of the momentum equation,

$$
\left(\frac{K_{y} l_{x}^{* 2}}{K_{x} H^{2}}\right)\left(\frac{\partial v^{\prime}}{\partial y^{\prime}} \frac{\partial x^{\prime}}{\partial y^{\prime}}\right)+\left(\frac{\partial v^{\prime}}{\partial x^{\prime}}\right)=\left(\frac{\partial T^{\prime}}{\partial x^{\prime}}\right) .
$$


Finally, the nondimensionalization of the energy equation (3), which is carried out in a similar way, leads to

$$
\begin{aligned}
& \left(\rho_{f} c_{p, f}\right)\left(\frac{u^{*}}{l_{x}^{*}}\right)\left(u^{\prime} \frac{\partial T^{\prime}}{\partial x^{\prime}}\right)+\left(\rho_{f} c_{p, f}\right)\left(\frac{v^{*}}{H}\right)\left(v^{\prime} \frac{\partial T^{\prime}}{\partial y^{\prime}}\right) \\
& =\frac{k_{m, x}}{l_{x}^{* 2}}\left(\frac{\partial^{2} T^{\prime}}{\partial x^{\prime 2}}\right)+\frac{k_{m, y}}{H^{2}}\left(\frac{\partial^{2} T^{\prime}}{\partial y^{\prime 2}}\right) .
\end{aligned}
$$

\section{Resulting Dimensionless Groups and Solutions}

The dimensionless groups that rule the steady state solution patterns of the problem must be investigated in light of the final dimensionless equations (14), (15), and (17). Starting with the first, the following monomial containing two unknowns $\left(l_{x}^{*}\right.$ and $\left.u^{*}\right)$ emerges:

$$
\pi_{1}=\left(\frac{\mu H u^{*}}{\left[\rho_{0} g \beta(\Delta T) K_{y}\right] l_{x}^{*}}\right) .
$$

The physical meaning of this monomial is clear since it comes from the mass conservation equation; it refers to the mass balance in steady state within the domain defined by the reference quantities $l_{x}^{*}$ and $H$, but we can investigate even deeper the approximate value of $\pi_{1}$. To do that, we estimate the order of magnitude $(\sim)$ of each derivative term appearing in (14). Making use of the range assigned above to the dimensionless variables, we can confirm that

$$
\left(\frac{\partial u^{\prime}}{\partial x^{\prime}}\right) \sim \frac{\Delta u^{\prime}}{\Delta x^{\prime}} \sim 1, \quad\left(\frac{\partial v^{\prime}}{\partial y^{\prime}}\right) \sim \frac{\Delta v^{\prime}}{\Delta y^{\prime}} \sim 1,
$$

a result that, when substituted in (14), provides the order of magnitude of the monomial

$$
\pi_{1}=\left(\frac{\mu H u^{*}}{\left[\rho_{0} g \beta(\Delta T) K_{y}\right] l_{x}^{*}}\right) \sim 1 .
$$

This means that, in terms of the reference quantities,

$$
\left(\frac{u^{*}}{l_{x}^{*}}\right) \sim\left(\frac{v^{*}}{H}\right)
$$

Let us continue with the dimensionless momentum of (16). This provides the new monomial

$$
\pi_{2}=\left(\frac{K_{y} l_{x}^{* 2}}{K_{x} H^{2}}\right)
$$

whose meaning is a kind of hydraulic permeability ratio corrected by the domain geometry parameters $l_{x}^{*}$ and $H$. It is interesting to note that the pure ratio $K_{x} / K_{y}$ is dimensionless from the classical point of view; however, when the discrimination is assumed, this ratio is not a dimensionless group. Besides, based on similar reasoning as regards the order of magnitude of $\pi_{2}$, it can immediately be asserted that

$$
\left(\frac{\partial v^{\prime}}{\partial y^{\prime}} \frac{\partial x^{\prime}}{\partial y^{\prime}}\right) \sim\left(\frac{\partial v^{\prime}}{\partial x^{\prime}}\right) \sim\left(\frac{\partial T^{\prime}}{\partial x^{\prime}}\right) \sim 1
$$

and, consequently,

$$
\pi_{2}=\left(\frac{K_{y} l_{x}^{* 2}}{K_{x} H^{2}}\right) \sim 1 .
$$

Finally, to derive the monomials of energy equation (17), we will divide all the terms by, for example, the last one $\left(k_{m, y} / H^{2}\right)$, rewriting the equation in the form

$$
\begin{aligned}
& \left(\frac{u^{*}}{l_{x}^{*}}\right)\left(\frac{\rho_{f} c_{p, f} H^{2}}{k_{m, y}}\right)\left(u^{\prime} \frac{\partial T^{\prime}}{\partial x^{\prime}}\right) \\
& +\left(\frac{v^{*}}{H}\right)\left(\frac{\rho_{f} c_{p, f} H^{2}}{k_{m, y}}\right)\left(v^{\prime} \frac{\partial T^{\prime}}{\partial y^{\prime}}\right) \\
& =\left(\frac{k_{m, x} H^{2}}{k_{m, y} l_{x}^{* 2}}\right)\left(\frac{\partial^{2} T^{\prime}}{\partial x^{\prime 2}}\right)+\left(\frac{\partial^{2} T^{\prime}}{\partial y^{\prime 2}}\right) .
\end{aligned}
$$

This provides three new dimensionless monomials

$$
\begin{gathered}
\pi_{3}=\left(\frac{u^{*}}{l_{x}^{*}}\right)\left(\frac{\rho_{f} c_{p, f} H^{2}}{k_{m, y}}\right), \quad \pi_{4}=\left(\frac{v^{*}}{H}\right)\left(\frac{\rho_{f} c_{p, f} H^{2}}{k_{m, y}}\right), \\
\pi_{5}=\left(\frac{k_{m, x} H^{2}}{k_{m, y} l_{x}^{* 2}}\right),
\end{gathered}
$$

two of which, $\pi_{3}$ and $\pi_{4}$, based on (21), are identical. Particularly, $\pi_{3}$ and $\pi_{4}$ are a kind of discriminated Rayleigh number. Besides, from the arguments used for the conservation and momentum equations, we can declare that these monomials must also be of the order of magnitude unity.

In short, the problem is ruled by three dimensionless groups, $\pi_{2}, \pi_{3}$ (or $\pi_{4}$ ), and $\pi_{5}$. Because of their properties of being dimensionless and of the order of magnitude unity, these monomials can be combined by mathematical manipulation and presented in different forms. Doing this, since we are interested in the unknown $l_{x}^{*}$ appearing in only one group for this variable to be expressed as a function of the other parameters, we will change the monomial $\pi_{5}$ for that resulting from the product $\pi_{5} \pi_{2}$, which does not contain $l_{x}^{*}$. The final renamed monomials are

$$
\begin{gathered}
\pi_{\mathrm{I}}=\left(\frac{K_{y} l_{x}^{* 2}}{K_{x} H^{2}}\right), \quad \pi_{\mathrm{II}}=\left(\frac{K_{y} k_{m, x}}{K_{x} k_{m, y}}\right), \\
\pi_{\mathrm{III}}=\left(\frac{\left[\rho_{0} g \beta(\Delta T) K_{y}\right] H}{\mu \alpha_{m, y}}\right),
\end{gathered}
$$

where $\alpha\left(\mathrm{m}^{2} / \mathrm{s}\right)$ is the thermal diffusivity and $\alpha_{m, y}=$ $k_{m, y} / \rho_{f} c_{p, f}$. Now, the solution for $l_{x}^{*}$ is

$$
l_{x}^{*}=H \sqrt{\frac{K_{x}}{K_{y}}} \Psi\left[\left(\frac{K_{y} k_{m, x}}{K_{x} k_{m, y}}\right),\left(\frac{\left[\rho_{0} g \beta(\Delta T) K\right] H}{\mu \alpha_{m, y}}\right)\right]
$$

with $\Psi$ an unknown function of the arguments $\pi_{\mathrm{II}}$ and $\pi_{\mathrm{III}}$. 
TABLE 1: Values of the physical parameters, S.I. (cases 1-12).

\begin{tabular}{|c|c|c|c|c|c|c|c|c|c|}
\hline & Case & $K_{x}$ & $K_{y}$ & $\alpha_{x}$ & $\alpha_{y}$ & $\mu$ & $\Delta \rho$ & $g$ & $v^{*}$ \\
\hline \multirow{5}{*}{ Group 1} & \multirow[t]{2}{*}{1} & $K_{x, 1}$ & $K_{y, 1}$ & $\alpha_{x, 1}$ & $\alpha_{y, 1}$ & $\mu_{1}$ & $\delta \rho_{1}$ & $g_{1}$ & \multirow[t]{2}{*}{$2.645 e-5$} \\
\hline & & $5.75 e-13$ & $2.3 e-12$ & $7.5 e-6$ & $3 e-5$ & $2 e-4$ & 230 & 10 & \\
\hline & \multirow[t]{2}{*}{2} & $3 K_{x, 1}$ & $3 K_{y, 1}$ & $2 \alpha_{x, 1}$ & $2 \alpha_{y, 1}$ & $1.5 \mu_{1}$ & $2 \delta \rho_{1}$ & $0.5 g_{1}$ & \multirow[t]{2}{*}{$5.29 e-5$} \\
\hline & & & $0.9 e-12$ & $1.3 e-5$ & $0 e-5$ & $3 e-4$ & 460 & & \\
\hline & 3 & $\begin{array}{c}0.5 K_{x, 1} \\
2.875 e-13\end{array}$ & $\begin{array}{c}0.5 K_{y, 1} \\
1.15 e-12\end{array}$ & $\begin{array}{c}1.5 \alpha_{x, 1} \\
1.125 e-5\end{array}$ & $\begin{array}{c}1.5 \alpha_{y, 1} \\
4.5 e-5\end{array}$ & $\begin{array}{c}2 \mu_{1} \\
4 e-4\end{array}$ & $\begin{array}{c}2 \delta \rho_{1} \\
460\end{array}$ & $\begin{array}{c}3 g_{1} \\
30\end{array}$ & $3.968 e-5$ \\
\hline \multirow{6}{*}{ Group 2} & \multirow{2}{*}{4} & $2 K_{x, 1}$ & $K_{y, 1}$ & $2 \alpha_{x, 1}$ & $\alpha_{y, 1}$ & $\mu_{1}$ & $\delta \rho_{1}$ & $g_{1}$ & \multirow{2}{*}{$2.645 e-5$} \\
\hline & & $1.15 e-12$ & $2.3 e-12$ & $1.5 e-5$ & $3 e-5$ & $2 e-4$ & 230 & 10 & \\
\hline & \multirow{2}{*}{5} & $4 K_{x, 1}$ & $2 K_{y, 1}$ & $4 \alpha_{x, 1}$ & $2 \alpha_{y, 1}$ & $2 \mu_{1}$ & $\delta \rho_{1}$ & $2 g_{1}$ & \multirow{2}{*}{$5.29 e-5$} \\
\hline & & $2.3 e-12$ & $4.6 e-12$ & $3 e-5$ & $6 e-5$ & $4 e-4$ & 230 & 20 & \\
\hline & \multirow[t]{2}{*}{6} & $K_{x, 1}$ & $0.5 K_{y, 1}$ & $\alpha_{x, 1}$ & $0.5 \alpha_{y, 1}$ & $0.5 \mu_{1}$ & $0.5 \delta \rho_{1}$ & $g_{1}$ & \multirow{2}{*}{$1.323 e-5$} \\
\hline & & $5.75 e-13$ & $1.15 e-12$ & $7.5 e-6$ & $1.5 e-5$ & $1 e-4$ & 115 & 10 & \\
\hline \multirow{6}{*}{ Group 3} & \multirow{2}{*}{7} & $5 K_{x, 1}$ & $K_{y, 1}$ & $5 \alpha_{x, 1}$ & $\alpha_{y, 1}$ & $\mu_{1}$ & $\delta \rho_{1}$ & $g_{1}$ & \multirow{2}{*}{$2.645 e-5$} \\
\hline & & $2.875 e-12$ & $2.3 e-12$ & $3.75 e-5$ & $3 e-5$ & $2 e-4$ & 230 & 10 & \\
\hline & \multirow{2}{*}{8} & $10 K_{x, 1}$ & $2 K_{y, 1}$ & $10 \alpha_{x, 1}$ & $2 \alpha_{y, 1}$ & $0.5 \mu_{1}$ & $0.5 \delta \rho_{1}$ & $g_{1}$ & \multirow{2}{*}{$5.29 e-5$} \\
\hline & & $5.75 e-12$ & $4.6 e-12$ & $7.5 e-5$ & $6 e-5$ & $1 e-4$ & 115 & 10 & \\
\hline & \multirow{2}{*}{9} & $2.5 K_{x, 1}$ & $0.5 K_{y, 1}$ & $25 \alpha_{x, 1}$ & $5 \alpha_{y, 1}$ & $0.5 \mu_{1}$ & $\delta \rho_{1}$ & $5 g_{1}$ & \multirow{2}{*}{$1.323 e-5$} \\
\hline & & $1.438 e-12$ & $1.15 e-12$ & $1.875 e-4$ & $1.5 e-4$ & $1 e-4$ & 230 & 50 & \\
\hline \multirow{6}{*}{ Group 4} & \multirow{2}{*}{10} & $K_{x, 1}$ & $3 K_{y, 1}$ & $\alpha_{x, 1}$ & $3 \alpha_{y, 1}$ & $\mu_{1}$ & $\delta \rho_{1}$ & $g_{1}$ & \multirow{2}{*}{$7.935 e-5$} \\
\hline & & $5.75 e-13$ & $6.9 e-12$ & $7.5 e-6$ & $9 e-5$ & $2 e-4$ & 230 & 10 & \\
\hline & \multirow{2}{*}{11} & $2 K_{x, 1}$ & $6 K_{y, 1}$ & $\alpha_{x, 1}$ & $3 \alpha_{y, 1}$ & $2 \mu_{1}$ & $2 \delta \rho_{1}$ & $0.5 g_{1}$ & \multirow{2}{*}{$7.935 e-5$} \\
\hline & & $1.15 e-12$ & $1.38 e-11$ & $7.5 e-6$ & $9 e-5$ & $4 e-4$ & 460 & 5 & \\
\hline & \multirow{2}{*}{12} & $(1 / 3) K_{x, 1}$ & $K_{y, 1}$ & $2 \alpha_{x, 1}$ & $6 \alpha_{y, 1}$ & $0.5 \mu_{1}$ & $\delta \rho_{1}$ & $3 g_{1}$ & \multirow{2}{*}{$1.587 e-4$} \\
\hline & & $1.92 e-13$ & $2.3 e-12$ & $1.5 e-5$ & $1.8 e-4$ & $1 e-4$ & 230 & 30 & \\
\hline
\end{tabular}

\section{Validation}

Several tests to validate the results of the above section are presented. These consist of a selection of scenarios (or cases) for which some of the parameters are suitably changed, retaining the same values for the final dimensionless groups $\left(\pi_{\mathrm{II}}\right.$ and $\left.\pi_{\mathrm{III}}\right)$ that rule the problem or determine its steady state (flow and temperature) patterns. The complexity of the problem requires numerical techniques for the solution and we have chosen the network method as the most suitable tool. This method whose basis can be found in GonzálezFernández [21] has been applied for the solution of a large number of nonlineal problems in different fields of science and engineering such as heat transfer [22], vibrations [23], electrochemistry [24], elasticity [25], and others [26]. The network model of the problem is run on the software Pspice [27]. For all simulations presented in this section, a grid of 80 (horizontal) $\times 20$ (vertical) volume elements was chosen .

For a better illustration of the results, the dependent variable velocity has been substituted by the stream function variable, $\psi\left(\mathrm{m}^{2} / \mathrm{s}\right)$; so, the steady state patterns of this variable directly show the way that fluid particles flow. The connection between velocity and stream function variables is given by the expressions

$$
u=\left(\frac{\partial \psi}{\partial y}\right), \quad v=-\left(\frac{\partial \psi}{\partial x}\right)
$$

The first simulation extends to twelve cases, for all of which the discriminate monomials $\pi_{\mathrm{II}}$ and $\pi_{\mathrm{III}}$, (27), retain the same value. They are organized, in turn, into four groups (three

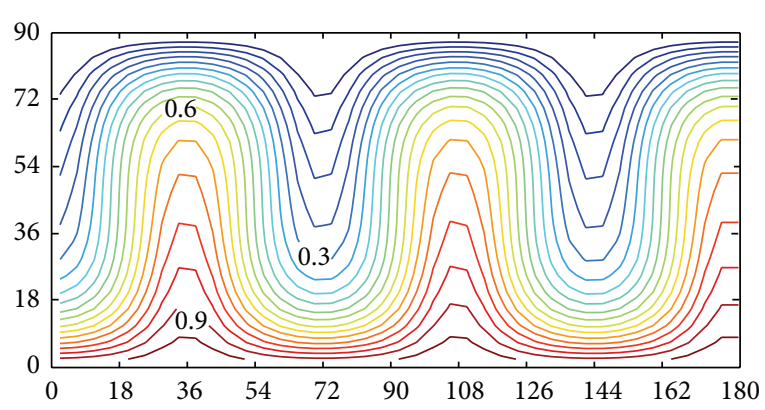

FIgURE 2: Temperature patterns for cases 1-3 (Group 1).

cases per group) in such a way that the parameters $H, K_{x}$, and $K_{y}$ are forced to change, retaining the same value for $H\left(K_{x} / K_{y}\right)^{1 / 2}$ in each group. In this form the characteristic length, given by (28), must have the same value for each group. Table 1 shows the value of the parameters of all cases in terms of their meaningful changes in relation to case 1 . The geometry of the scenario is $L=360(\mathrm{~m})$ and $H=90(\mathrm{~m})$, while the temperatures are $T_{b}=1^{\circ} \mathrm{C}, T_{u}=0^{\circ} \mathrm{C}$; finally, the stream function at all boundaries has the constant value $\psi_{b}=\psi_{u}=\psi_{l}=\psi_{r}=0$, allowing the impermeability condition to be applied.

With the values of Table 1 , where $\alpha=k_{m} / \rho_{f} c_{p, f}$ is the thermal diffusivity, $\pi_{\mathrm{II}}=1$ and $\pi_{\mathrm{III}}=79.4$ for all cases (the high value of $\pi_{\mathrm{III}}$ will be justified later). As regards $\pi_{\mathrm{I}}$, we expect it to retain the same value in all cases and its order of magnitude to be unity.

Figures 2 and 3 show the temperature and stream function patterns for the cases 1 to 3 (Group 1). As expected, the 

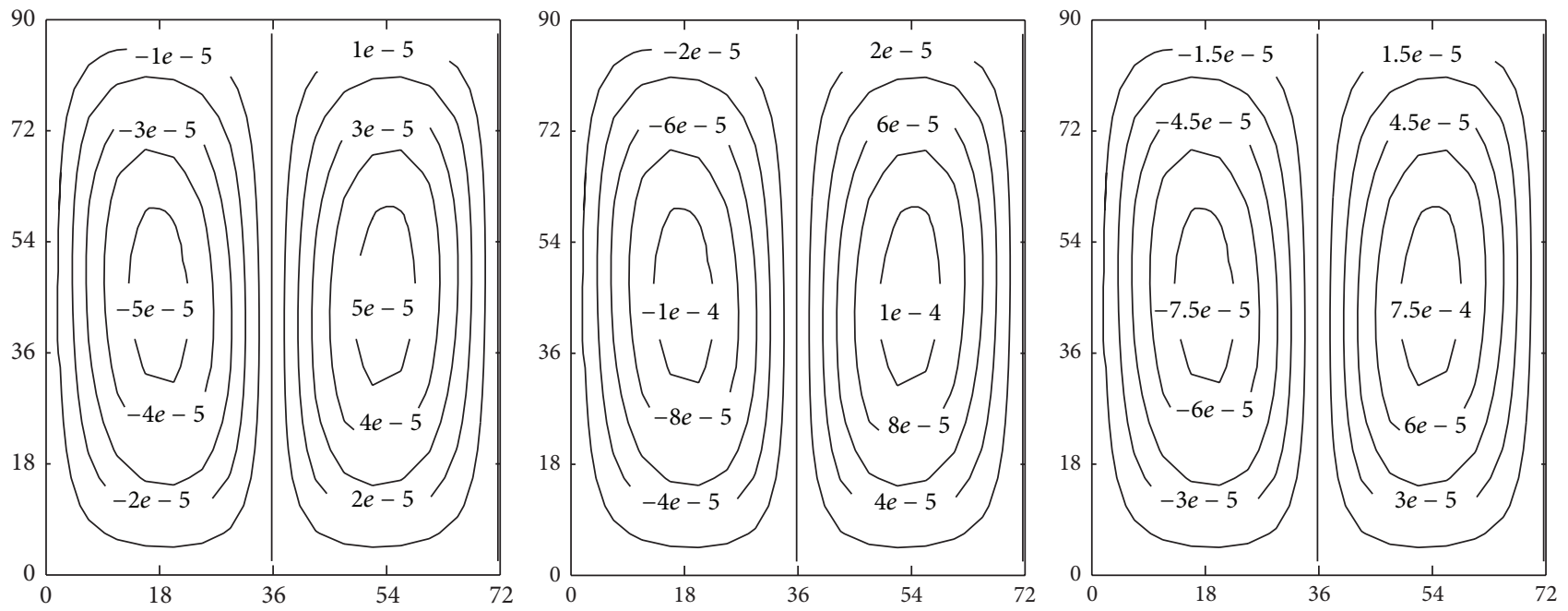

Figure 3: Stream function pattern for cases 1-3 (Group 1).

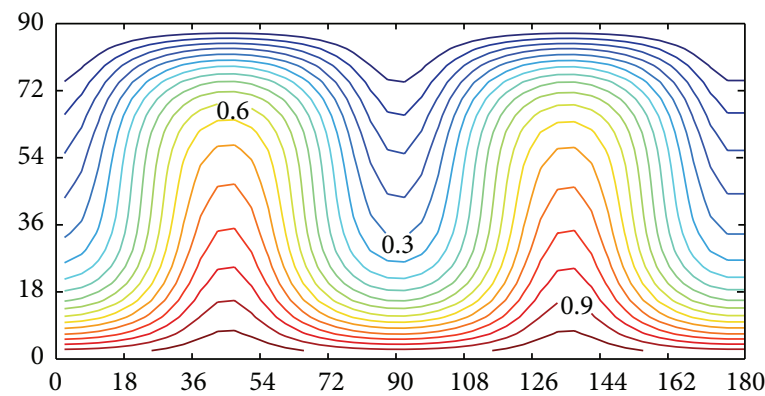

FIgURE 4: Temperature patterns for cases 4-6 (Group 2).
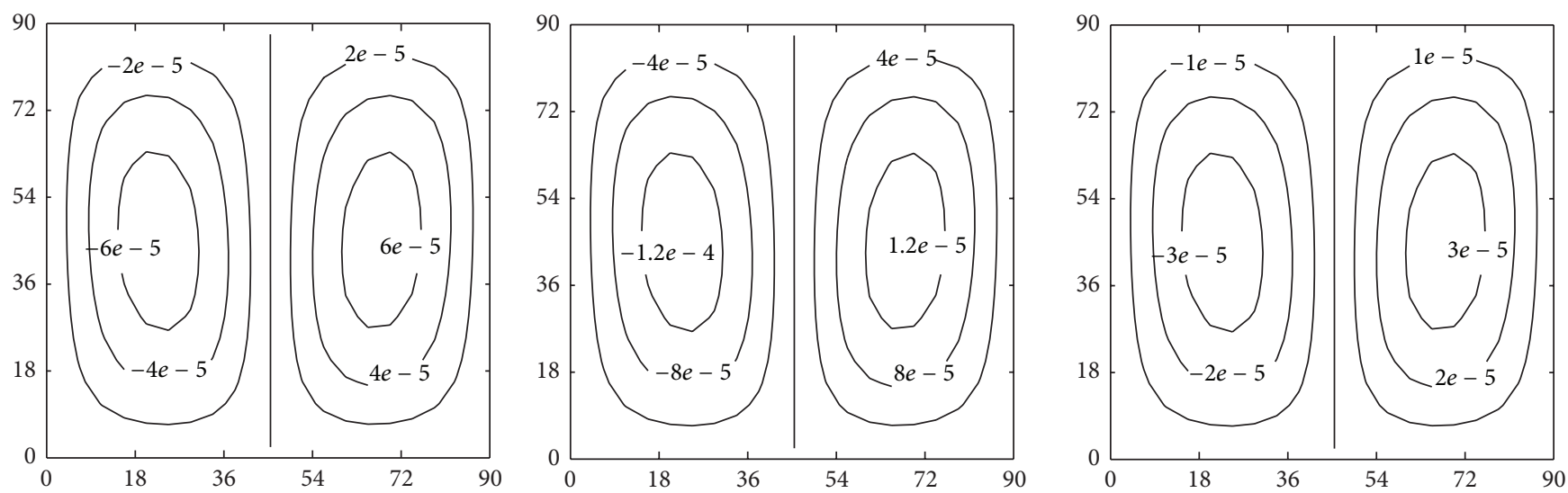

FIgURE 5: Stream function pattern for cases 4-6 (Group 2).

temperature patterns, which extend to half of the domain, are identical for the three cases; also, the stream function patterns, which extend to the two cells adjoining the left side, are identical in aspect, but their values of the stream function are proportional to the Darcy velocity, a parameter that differs for each case (Table 1).

Similar results, reflecting the expected patterns, are obtained by numerical simulation for the three other groups.
On the one hand, Figures 4, 6, and 8 show the temperature patterns for Groups 2 (cases 4-6), 3 (cases 7-9), and 4 (cases 10-12), respectively; in all the simulations identical patterns are obtained for the three cases of each group. On the other hand, Figures 5, 7, and 9 show the stream function patterns of the respective groups; as occurring in the cases of Group 1 , these patterns retain the same aspect, but their stream function values once again depend on the Darcy velocity. 


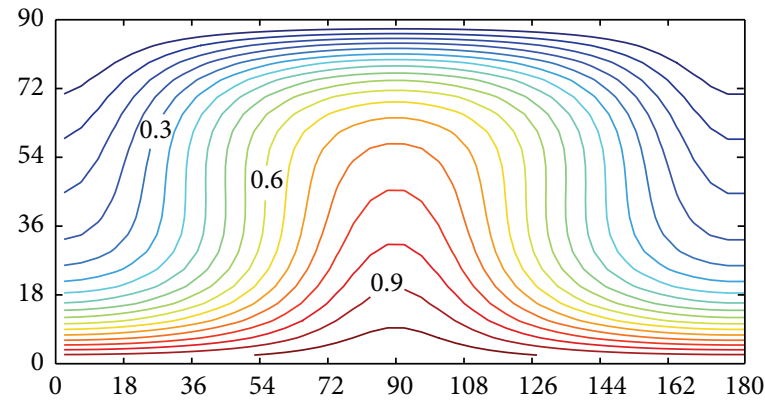

FIgURE 6: Temperature patterns for cases 7-9 (Group 3).
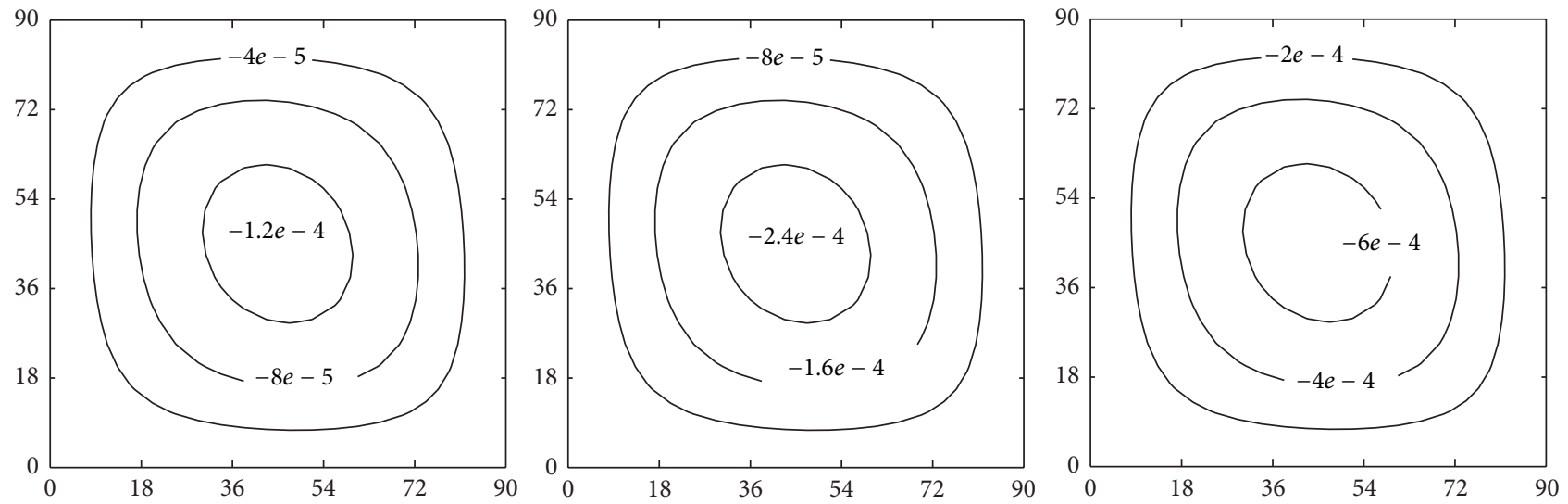

FIgURE 7: Stream function pattern for cases 7-9 (Group 3).

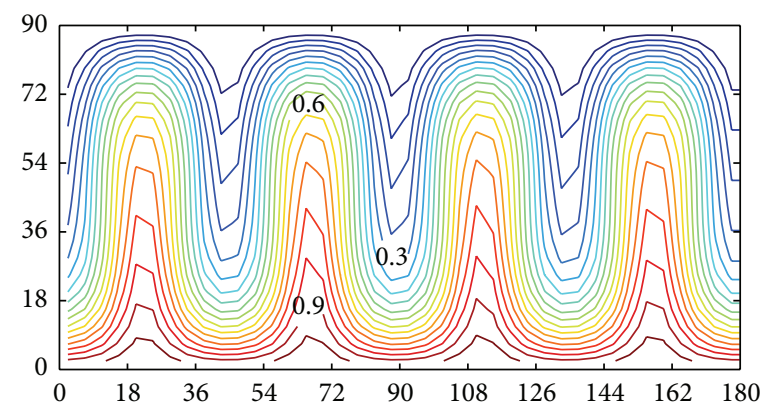

Figure 8: Temperature patterns for cases 10-12 (Group 4).

TABLE 2: Number of cells and characteristic lengths for cases 1-12.

\begin{tabular}{lcccc}
\hline Group & 1 & 2 & 3 & 4 \\
\hline$H\left(K_{x} / K_{y}\right)^{1 / 2}$ & 45 & 63.64 & 100.62 & 25.98 \\
Number of cells & 10 & 8 & 4 & 16 \\
$l^{*}{ }_{x}$ & 36 & 45 & 90 & 22.5 \\
$\Psi\left[\pi_{\mathrm{II}}, \pi_{\mathrm{III}}\right]=\pi_{\mathrm{I}}$ & 0.87 & 0.71 & 0.89 & 0.87 \\
\hline
\end{tabular}

Note that cases 10 and 11 (of group 4) have the same stream function pattern due to their identical Darcy velocity.

Table 2 shows the number of cells and the characteristic length resulting from each simulation. Since the monomials $\pi_{\text {II }}$ and $\pi_{\text {III }}$ retain the same value (79.35 and 1 , resp.) for all cases, the value of the unknown function $\Psi\left[\pi_{\mathrm{II}}, \pi_{\mathrm{III}}\right]$ must also be the same and this, according to (28), provides the same value for $l_{x}^{*}$ in each group. In turn, within each group, the factor $H\left(K_{x} / K_{y}\right)^{1 / 2}$ remains constant and, as a consequence, both the characteristic length and the number of cells of the domain must also retain a constant value. A fundamental result of discrimination is that the unknown function of the arguments formed by the dimensionless groups of a problem, except for the group that contains the unknown variable in which we are interested, has an order of magnitude unity regardless of the particular values of the arguments. The numerical simulations confirm this result: 

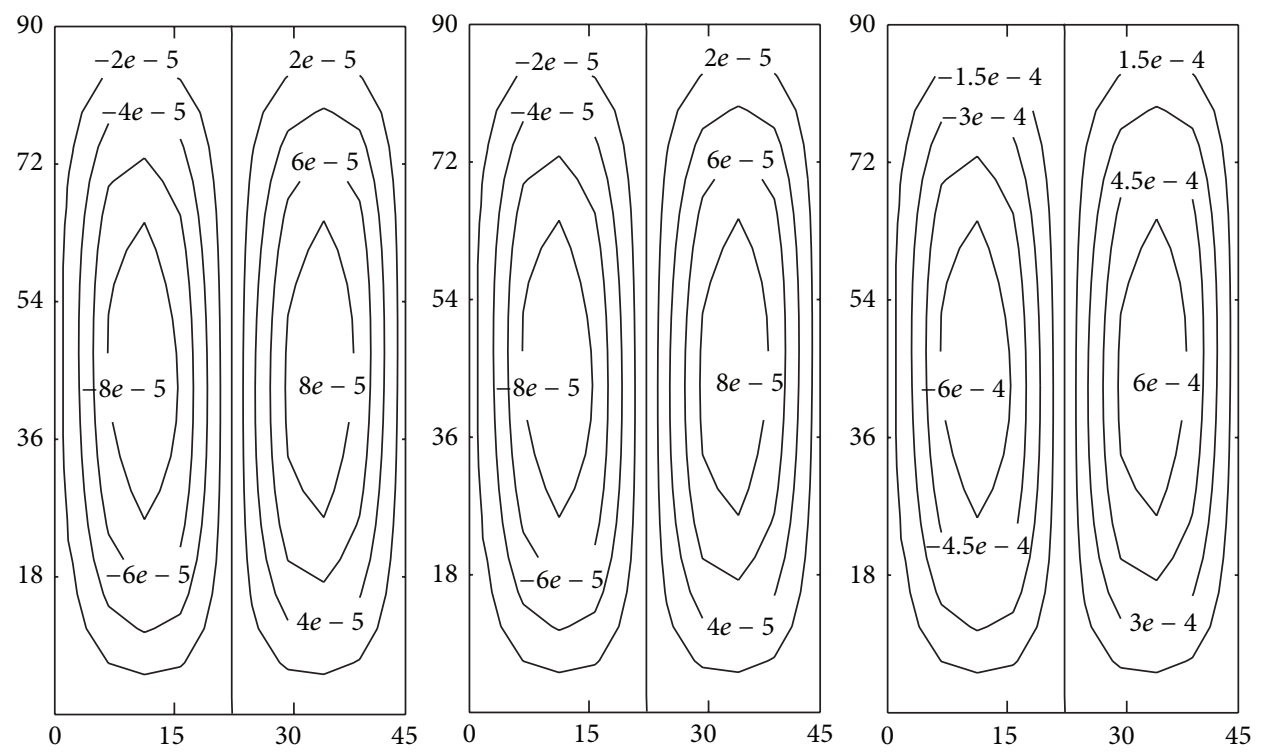

FIgURE 9: Stream function pattern for cases 10-12 (Group 4).

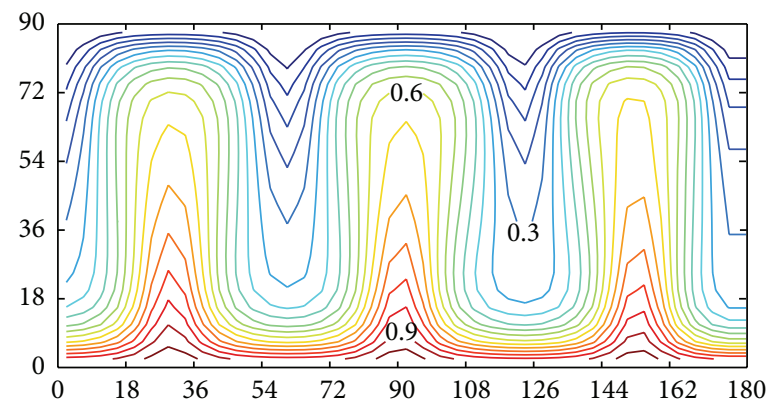

FIgURE 10: Temperature patterns for cases 13-14 (Group 5).
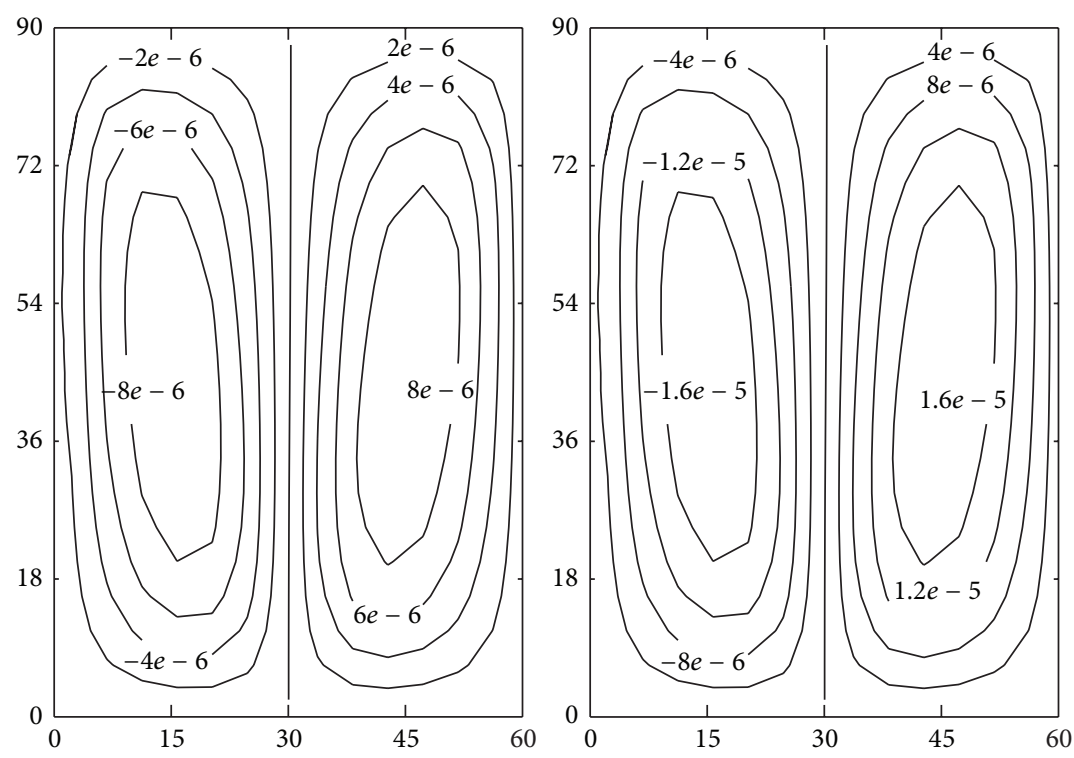

FIGURE 11: Stream function pattern for cases 13-14 (Group 5). 


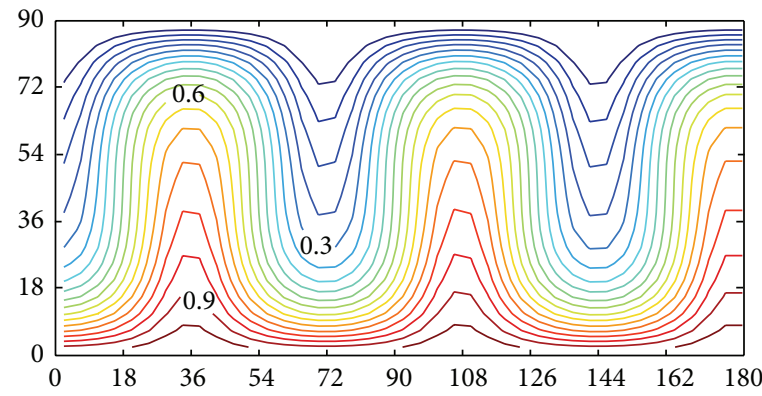

FIgURE 12: Temperature patterns for cases 15-16 (Group 6).
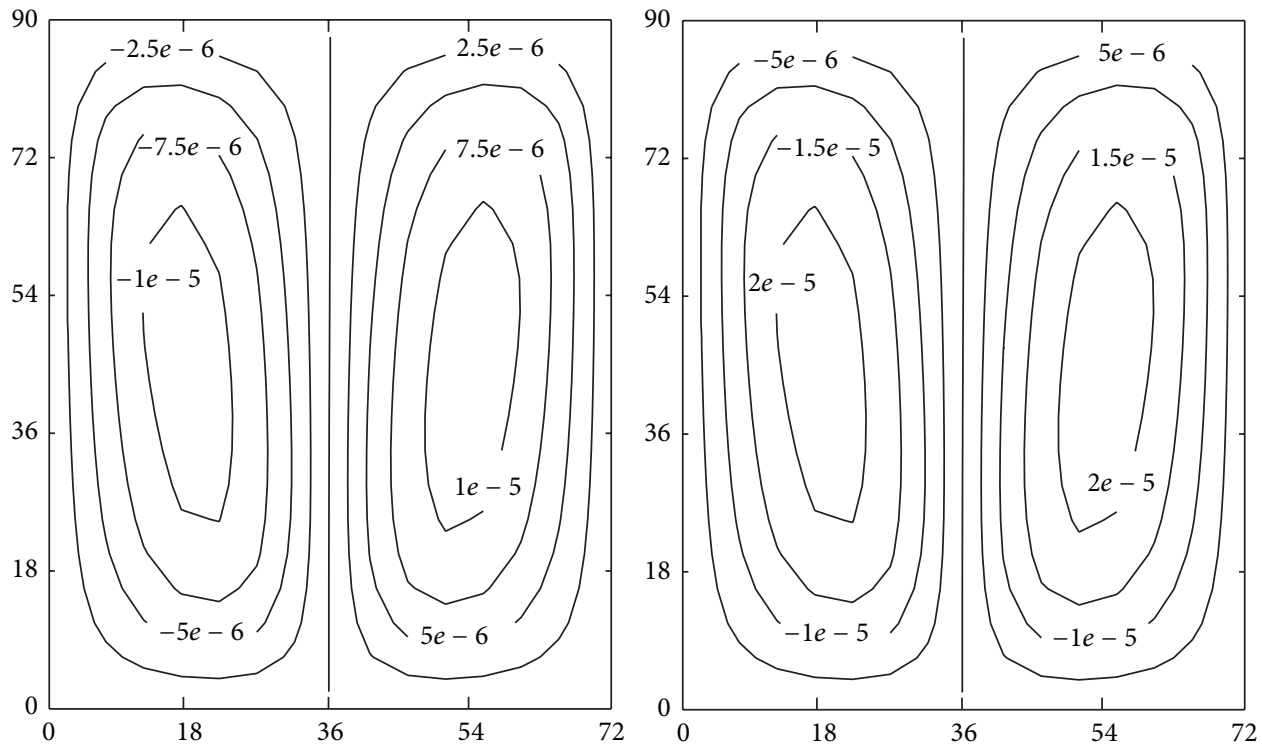

FIGURE 13: Stream function pattern for cases 15-16 (Group 6).

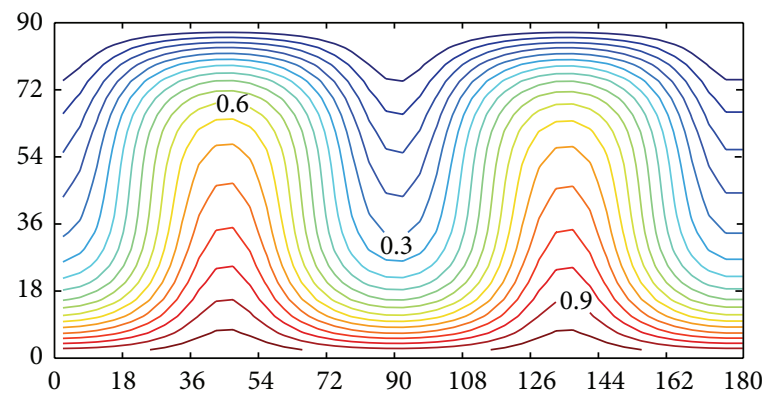

FIGURE 14: Temperature patterns for cases 17-18 (Group 7).

$\Psi\left(\pi_{\mathrm{II}}=79.35, \pi_{\mathrm{III}}=1\right)=0.8,0.71,0.89$, and 0.87 (all of the order of magnitude unity) for Groups 1, 2, 3, and 4, respectively.

The second simulation extends to ten cases for which $\pi_{\mathrm{II}}=165.6$ and $\pi_{\mathrm{III}}=1$. These are organized in five groups (two cases for each group) with constant values for the factor $H\left(K_{x} / K_{y}\right)^{1 / 2}$ within each group. The scenario has the same geometry and boundary conditions as those of the first simulation. Table 3 shows the parameters for all cases and any meaningful change compared with case 13 .

Figures 10 and 11 show the temperature and stream function patterns for cases 13 to 14 (Group 5). Again, as expected, the temperature patterns are the same, while the stream function patterns, despite retaining the same aspect, 

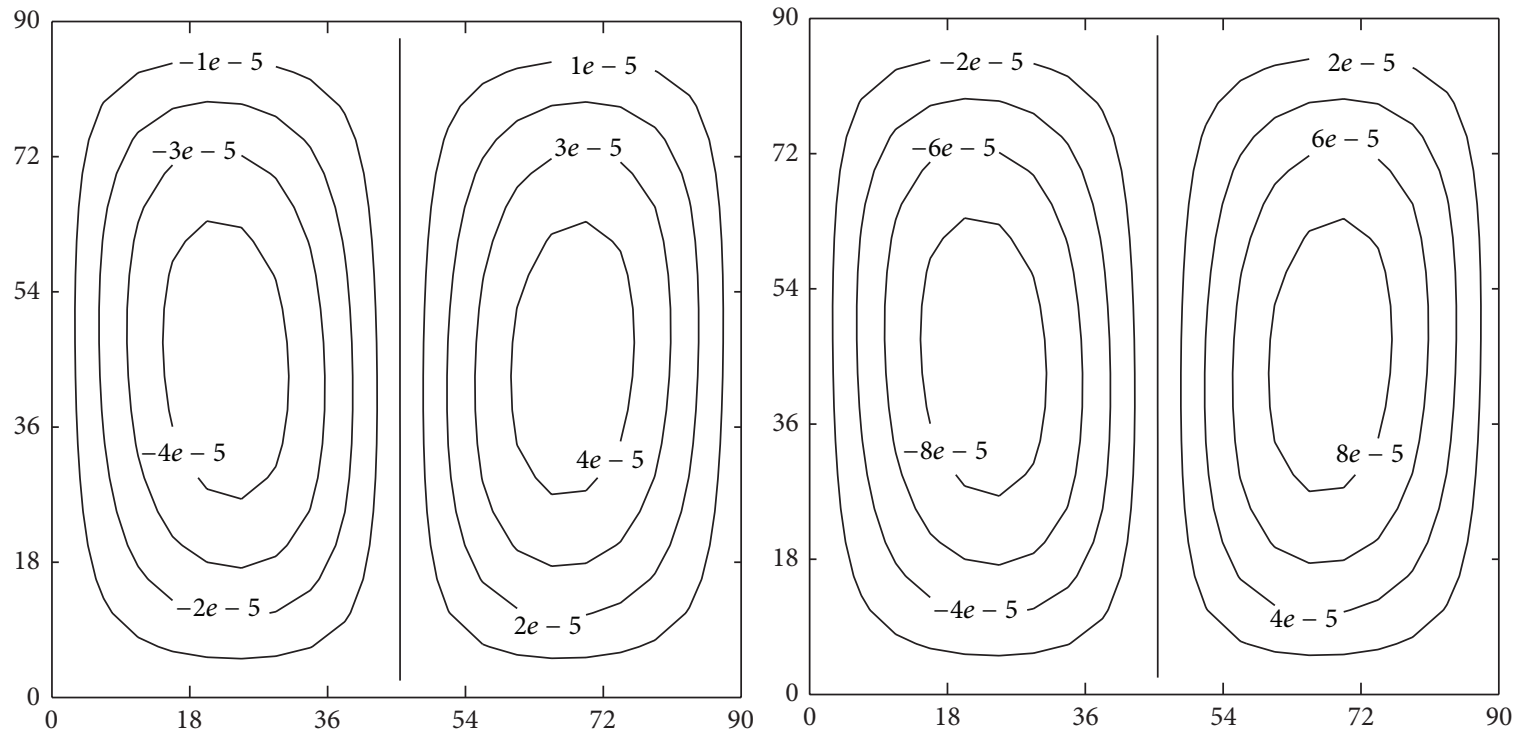

FIGURE 15: Stream function pattern for cases 17-18 (Group 7).

TABLE 3: Values of the physical parameters, S.I. (cases 13-22).

\begin{tabular}{|c|c|c|c|c|c|c|c|c|c|}
\hline & Case & $K_{x}$ & $K_{y}$ & $\alpha_{x}$ & $\alpha_{y}$ & $\mu$ & $\Delta \rho$ & $g$ & $v^{*}$ \\
\hline \multirow{2}{*}{ Group 5} & 13 & $\begin{array}{c}K_{x, 13} \\
1.94 e-13\end{array}$ & $\begin{array}{c}K_{y, 13} \\
4.8 e-13\end{array}$ & $\begin{array}{c}\alpha_{x, 13} \\
1.21 e-6\end{array}$ & $\begin{array}{c}\alpha_{y, 13} \\
3 e-6\end{array}$ & $\begin{array}{c}\mu_{13} \\
2 e-4\end{array}$ & $\begin{array}{l}\Delta \rho_{13} \\
230\end{array}$ & $\begin{array}{c}g_{13} \\
10\end{array}$ & $5.520 e-6$ \\
\hline & 14 & $\begin{array}{c}K_{x, 13} \\
194 e-13\end{array}$ & $\begin{array}{c}K_{y, 13} \\
4.8 e-13\end{array}$ & $\begin{array}{c}2 \alpha_{x, 13} \\
242 e-6\end{array}$ & $\begin{array}{l}2 \alpha_{y, 13} \\
6 e-6\end{array}$ & $\begin{array}{l}0.5 \mu_{13} \\
1 e-4\end{array}$ & $\begin{array}{c}0.5 \Delta \rho_{13} \\
115\end{array}$ & $\begin{array}{c}2 g_{13} \\
20\end{array}$ & $1.104 e-5$ \\
\hline \multirow{2}{*}{ Group 6} & 15 & $\begin{array}{c}\sqrt{2 K_{x, 13}} \\
2.743 e-13\end{array}$ & $\begin{array}{c}K_{y, 13} \\
4.8 e-13\end{array}$ & $\begin{array}{c}\sqrt{2 \alpha_{x, 13}} \\
1.711 e-6\end{array}$ & $\begin{array}{c}\alpha_{y, 13} \\
3 e-6\end{array}$ & $\begin{array}{c}\mu_{13} \\
2 e-4\end{array}$ & $\begin{array}{c}\Delta \rho_{13} \\
230\end{array}$ & $\begin{array}{l}g_{13} \\
10\end{array}$ & $5.520 e-6$ \\
\hline & 16 & $\begin{array}{c}\sqrt{2 K_{x, 13}} \\
2.744 e-13\end{array}$ & $\begin{array}{c}K_{y, 13} \\
4.8 e-13\end{array}$ & $\begin{array}{c}2 \sqrt{2 \alpha_{x, 13}} \\
3.422 e-6\end{array}$ & $\begin{array}{l}2 \alpha_{y, 13} \\
6 e-6\end{array}$ & $\begin{array}{l}0.5 \mu_{13} \\
1 e-4\end{array}$ & $\begin{array}{c}0.5 \Delta \rho_{13} \\
115\end{array}$ & $\begin{array}{c}2 g_{13} \\
20\end{array}$ & $1.104 e-5$ \\
\hline \multirow{2}{*}{ Group 7} & 17 & $\begin{array}{c}2 K_{x, 13} \\
3.886 e-13\end{array}$ & $\begin{array}{c}K_{y, 13} \\
4.8 e-13\end{array}$ & $\begin{array}{c}2 \alpha_{x, 13} \\
2.425 e-6\end{array}$ & $\begin{array}{c}\alpha_{y, 13} \\
3 e-6\end{array}$ & $\begin{array}{c}\mu_{13} \\
2 e-4\end{array}$ & $\begin{array}{c}\Delta \rho_{13} \\
230\end{array}$ & $\begin{array}{c}g_{13} \\
10\end{array}$ & $5.520 e-6$ \\
\hline & 18 & $\begin{array}{c}2 K_{x, 13} \\
3.886 e-13\end{array}$ & $\begin{array}{c}K_{y, 13} \\
4.8 e-13\end{array}$ & $\begin{array}{c}4 \alpha_{x, 13} \\
4.85 e-6\end{array}$ & $\begin{array}{l}2 \alpha_{y, 13} \\
6 e-6\end{array}$ & $\begin{array}{l}0.5 \mu_{13} \\
1 e-4\end{array}$ & $\begin{array}{c}0.5 \Delta \rho_{13} \\
115\end{array}$ & $\begin{array}{c}2 g_{13} \\
20\end{array}$ & $1.104 e-5$ \\
\hline \multirow{2}{*}{ Group 8} & 19 & $\begin{array}{c}4 K_{x, 13} \\
7.772 e-13\end{array}$ & $\begin{array}{c}K_{y, 13} \\
4.8 e-13\end{array}$ & $\begin{array}{c}4 \alpha_{x, 13} \\
4.85 e-6\end{array}$ & $\begin{array}{c}\alpha_{y, 13} \\
3 e-6\end{array}$ & $\begin{array}{c}\mu_{13} \\
2 e-4\end{array}$ & $\begin{array}{c}\Delta \rho_{13} \\
230\end{array}$ & $\begin{array}{l}g_{13} \\
10\end{array}$ & $5.520 e-6$ \\
\hline & 20 & $\begin{array}{c}4 K_{x, 13} \\
7.772 e-13\end{array}$ & $\begin{array}{c}K_{y, 13} \\
4.8 e-13\end{array}$ & $\begin{array}{c}8 \alpha_{x, 13} \\
9.7 e-6\end{array}$ & $\begin{array}{l}2 \alpha_{y, 13} \\
6 e-6\end{array}$ & $\begin{array}{l}0.5 \mu_{13} \\
1 e-4\end{array}$ & $\begin{array}{c}0.5 \Delta \rho_{13} \\
115\end{array}$ & $\begin{array}{c}2 g_{13} \\
20\end{array}$ & $1.104 e-5$ \\
\hline \multirow{2}{*}{ Group 9} & 21 & $\begin{array}{c}0.5 K_{x, 13} \\
9.715 e-14\end{array}$ & $\begin{array}{c}K_{y, 13} \\
4.8 e-13\end{array}$ & $\begin{array}{c}0.5 \alpha_{x, 13} \\
6.0625 e-7\end{array}$ & $\begin{array}{c}\alpha_{y, 13} \\
3 e-6\end{array}$ & $\begin{array}{c}\mu_{13} \\
2 e-4\end{array}$ & $\begin{array}{c}\Delta \rho_{13} \\
230\end{array}$ & $\begin{array}{c}g_{13} \\
10\end{array}$ & $5.520 e-6$ \\
\hline & 22 & $\begin{array}{c}0.5 K_{x, 13} \\
9.715 e-14\end{array}$ & $\begin{array}{c}K_{y, 13} \\
4.8 e-13\end{array}$ & $\begin{array}{c}\alpha_{x, 13} \\
1.21 e-6\end{array}$ & $\begin{array}{l}2 \alpha_{y, 13} \\
6 e-6\end{array}$ & $\begin{array}{l}0.5 \mu_{13} \\
1 e-4\end{array}$ & $\begin{array}{c}0.5 \Delta \rho_{13} \\
115\end{array}$ & $\begin{array}{c}2 g_{13} \\
20\end{array}$ & $1.104 e-5$ \\
\hline
\end{tabular}

TABLE 4: Number of cells and characteristic lengths for cases 13-22.

\begin{tabular}{lccccc}
\hline Group & 5 & 6 & 7 & 8 & 9 \\
\hline$H\left(K_{x} / K_{y}\right)^{1 / 2}$ & 57.26 & 68.043 & 80.98 & 114.52 & 40.49 \\
Number of cells & 12 & 10 & 8 & 6 & 16 \\
$l^{*}{ }_{x}$ & 30 & 36 & 45 & 60 & 22.5 \\
$\Psi\left[\pi_{\mathrm{II}}, \pi_{\mathrm{III}}\right]=\pi_{\mathrm{I}}$ & 0.524 & 0.529 & 0.556 & 0.524 & 0.556 \\
\hline
\end{tabular}

provide a net flow velocity proportional to the Darcy velocity. The patterns of the rest of the groups are shown in Figures 1213 (Group 6), Figures 14-15 (Group 7), Figures 16-17 (Group 8), and Figures 18-19 (Group 9).
Table 4 organizes the rest of the information: characteristic length, total number of cells, factor $H\left(K_{x} / K_{y}\right)^{1 / 2}$, and $\Psi\left[\pi_{\mathrm{II}}, \pi_{\mathrm{III}}\right]$. It is interesting to note that, despite the appreciable change in the monomial $\pi_{2}$ from 79.35 to 165.6 , the value of the function $\Psi\left[\pi_{\mathrm{II}}, \pi_{\mathrm{III}}\right]$ scarcely changes from approximately 0.80 (for $\pi_{2}=79.35$ ) to 0.55 (for $\pi_{2}=165.6$ ), determining the same order of magnitude for the monomial $\pi_{\mathrm{I}}$. Of course $l_{x}^{*}$ depends on the factor $H\left(K_{x} / K_{y}\right)^{1 / 2}$ and has the same value for both cases of each group.

A comparison between the results for Groups 2 and 6 confirms the relatively small influence of the monomial $\pi_{2}$ 


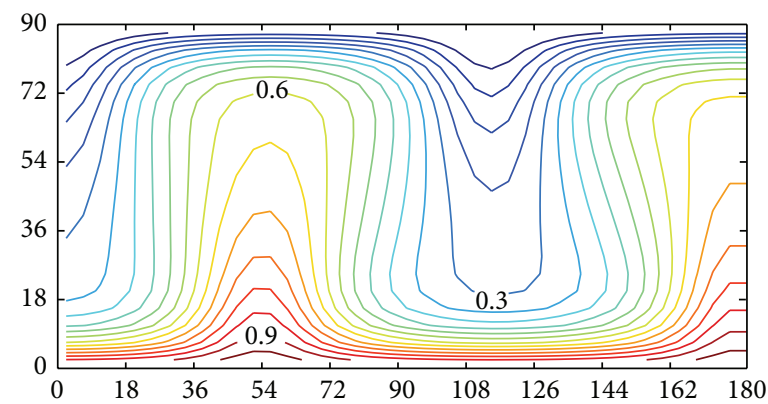

FIGURE 16: Temperature patterns for cases 19-20 (Group 8).

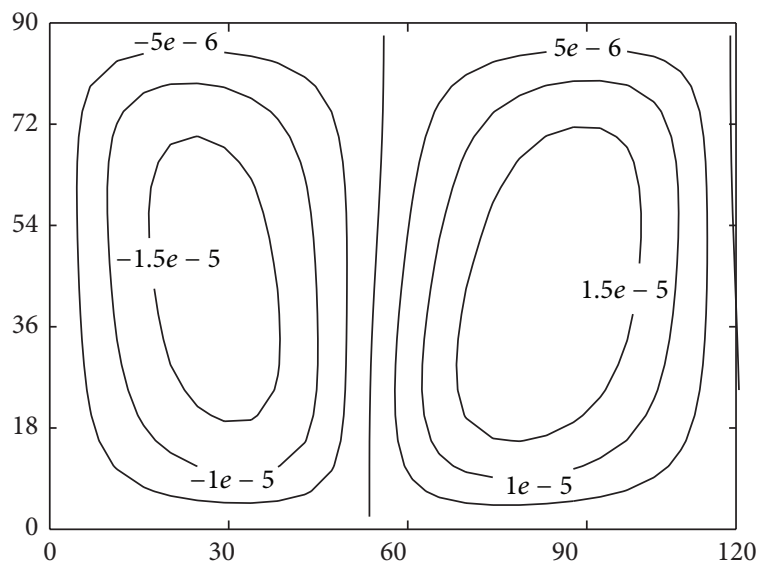

FIGURE 17: Stream function pattern for cases 19-20 (Group 8).

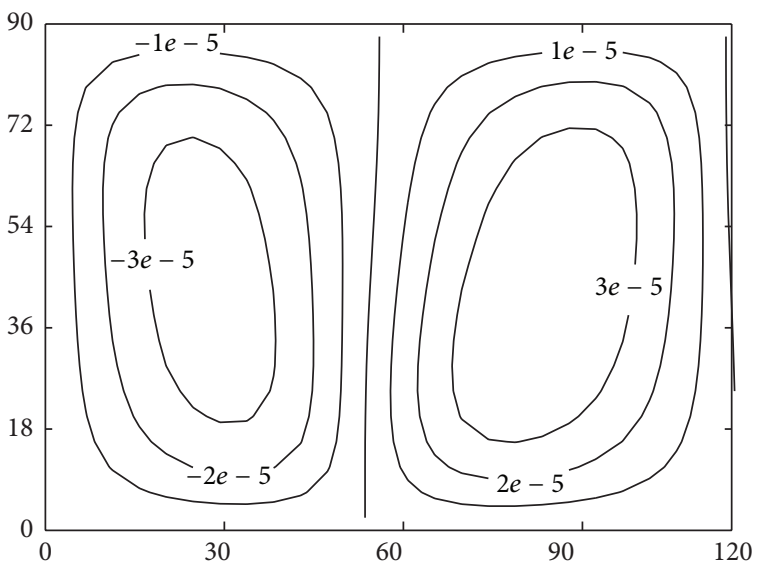

(a kind of discriminated Rayleigh number) in the length of the cell, compared with the appreciable change in $\pi_{2}$. While $\pi_{2}$ changes from 79.35 to $165.6, l_{x}^{*}$ changes from 45 to $36 \mathrm{~m}$.

To finish, we will return to the question why we assumed large values for $\pi_{2}$ (the discriminated Ra number). Although the scientific literature for isotropic scenarios uses a wide range of values (from $\approx 40$ to more than 1000 ; indeed, convection in isotropic media takes starts around $\pi_{2}=40$ ), according to the formal protocol used for the derivation of the dimensionless groups, these values should be of the order of magnitude unity. Further investigations are needed to throw light on this point.

In complex coupled problems such as the application studied in this paper, besides the global balances occurring in the complete domain, new balances could take place in small regions or subdomains, in which neither the geometrical parameters nor the governing equations are necessarily the same as those that rule the global problem. While the governing equations of a subdomain are a simplification of the governing equations of the complete problem and cannot provide new dimensionless numbers through nondimensionalization, the geometrical parameters (the fractions of the total lengths that define the subdomain) should reduce the order of magnitude of a monomial from its high value (when referring to the complete domain) to unity (when applied to a suitable subdomain), especially if the geometrical parameters appear in the monomial as a potential dependence. Indeed, this is the most probable explanation to justify the high controversial value of $\pi_{2}$ in this problem.

\section{Conclusions}

Despite the fact that it is currently applied by researchers to investigate the dimensionless groups that determine the solution patterns of a given problem, classical nondimensionalization may not lead to the most precise solution, that is, to the expressions and number of dimensionless groups that independently rule the solution, particularly in complex problems. This is the case, for example, in conjugate processes with anisotropic domains. In these cases, we have to take into account the use of discrimination in the process of nondimensionalization. The formal application of this technique considers the quantities of vectorial character (lengths, velocities, etc.) as if they were independent of each spatial direction and uses suitable references (one for each spatial direction) to make each one of the dependent and independent variables of the problem dimensionless. In addition, the values of the references must allow the variables to range in the interval $[0,1]$. If no information is given at the statement of the problem regarding one of these references, it must be established as if the references were known. 


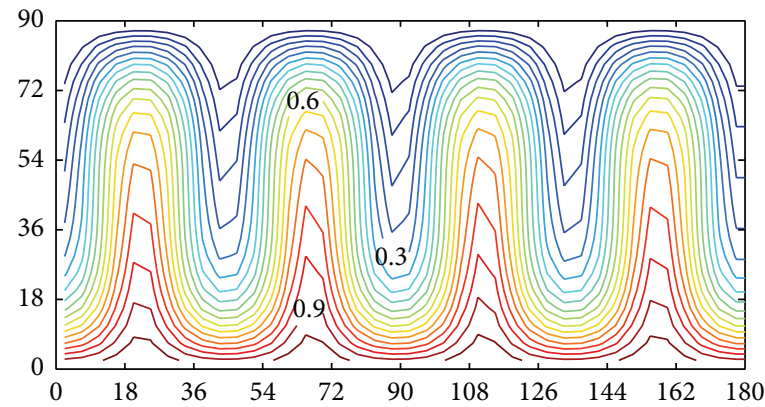

FIgURE 18: Temperature patterns for cases 21-22 (Group 9).
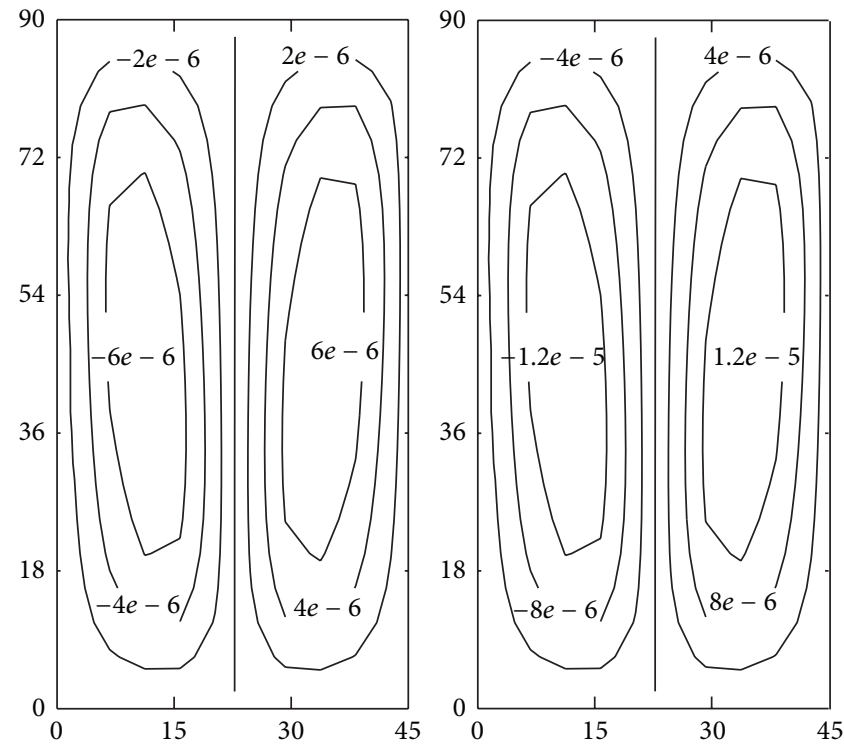

FIGURE 19: Stream function pattern for cases 21-22 (Group 9).

By doing this, the resulting groups, besides ruling the solution of the problem, have two interesting inherent properties: they are of the order of magnitude unity and have a clear physical meaning in terms of balance of the quantities that balance in the process. In addition, since, in terms of the dimensionless groups, the solution can be expressed by putting one group in terms of the rest by means of an unknown function of their arguments, it is always possible to determine the order of magnitude of the above-mentioned unknown reference.

In the context of the problem of natural convection between horizontal plates heated from below, in a hydraulic and thermal anisotropic medium, three dimensionless groups emerged that could not be obtained without discrimination. The order of magnitude of the characteristic length of the typical cell is directly derived from these groups.

\section{Conflict of Interests}

The authors declare that there is no conflict of interests regarding the publication of this paper.

\section{References}

[1] J. Palacios, Dimensional Analysis, Macmillan, London, UK, 1964.

[2] T. Szirtes, Applied Dimensional Analysis, McGraw-Hill, New York, NY, USA, 1997.

[3] A. A. Sonin, The Physical Basis of Dimensional Analysis, vol. 57, Department of Mechanical Engineering, Massachusetts Institute of Technology, Cambridge, Mass, USA, 2001.

[4] E. R. van Driest, "On dimensional analysis and the presentation of data fluid flow problems," Journal of Applied Mechanics, pp. 34-40, 1947.

[5] P. H. Schultz, "Atmospheric effects on ejecta emplacement," Journal of Geophysical Research, vol. 97, no. 7, pp. 11-662, 1992.

[6] R. Hilfer and R. Helmig, "Dimensional analysis and upscaling of two-phase flow in porous media with piecewise constant heterogeneities," Advances in Water Resources, vol. 27, no. 10, pp. 1033-1040, 2004.

[7] J. J. Rajesh and J. Bijwe, "Dimensional analysis for abrasive wear behaviour of various polyamides," Tribology Letters, vol. 18, no. 3, pp. 331-340, 2005.

[8] T. C. Chuang, "Dimensional analysis for Gorter-Mellink counter flow convection in pressurized superfluid helium," 
International Journal of Heat and Mass Transfer, vol. 48, no. 2, pp. 455-459, 2005.

[9] J. Hristov, "Magnetic field assisted fluidization-dimensional analysis addressing the physical basis," China Particuology, vol. 5, no. 1-2, pp. 103-110, 2007.

[10] C. N. Madrid and F. Alhama, "Discrimination: a fundamental and necessary extension of classical dimensional analysis theory," International Communications in Heat and Mass Transfer, vol. 33, no. 3, pp. 287-294, 2006.

[11] J. I. Prieto, J. Fano, R. Diaz, and M. A. Gonzalez, "Application of discriminated dimensional analysis to the kinematic Stirling engine," Journal of Mechanical Engineering Science, vol. 208, no. 5, pp. 347-353, 1994.

[12] V. D. Zimparov and V. M. Petkov, "Application of discriminated analysis to low Reynolds number swirl flows in circular tubes with twisted-tape inserts. Pressure drop correlations," International Review of Chemical Engineering, vol. 1, no. 4, pp. 346-356, 2009.

[13] M. Capobianchi and A. Aziz, "A scale analysis for natural convective flows over vertical surfaces," International Journal of Thermal Sciences, vol. 54, pp. 82-88, 2012.

[14] S. Middelman, An Introduction to Heat and Mass Transfer, John Wiley \& Sons, New York, NY, USA, 1998.

[15] A. J. Smits, A Physical Introduction to Fluids Mechanics, John Wiley \& Sons, New York, NY, USA, 2000.

[16] Y. A. Cengel, Heat Transfer. A Practical Approach, McGraw-Hill, New York, NY, USA, 2nd edition, 2004.

[17] C. N. Madrid and F. Alhama, "Discriminated dimensional analysis of the energy equation: application to laminar forced convection along a flat plate," International Journal of Thermal Sciences, vol. 44, no. 4, pp. 333-341, 2005.

[18] C. N. Madrid and F. Alhama, "Study of the laminar natural convection problem along an isothermal vertical plate based on discriminated dimensional analysis," Chemical Engineering Communications, vol. 195, no. 12, pp. 1524-1537, 2008.

[19] E. Holzbecher and Y. Yusa, "Numerical experiments on free and forced convection in porous media," International Journal of Heat and Mass Transfer, vol. 38, no. 11, pp. 2109-2115, 1995.

[20] E. Holzbecher, Modelling Density-Driven Flow in Porous Media, Springer, Berlin, Germany, 1998.

[21] C. F. González-Fernández, "Applications of the network simulation method to transport processes," in Network Simulation Method, J. Horno, Ed., Research Signpost, Trivandrum, India, 2002.

[22] F. Illán and M. Alarcón, "Numerical analysis of combustion and transient heat transfer processes in a two-stroke SI engine," Applied Thermal Engineering, vol. 30, no. 16, pp. 2469-2475, 2010.

[23] E. Castro, M. T. García-Hernández, and A. Gallego, “Transversal waves in beams via the network simulation method," Journal of Sound and Vibration, vol. 283, no. 3-5, pp. 997-1013, 2005.

[24] J. J. López-García, C. Grosse, and J. Horno, "Numerical study of colloidal suspensions of soft spherical particles using the network method: 2. AC electrokinetic and dielectric properties," Journal of Colloid and Interface Science, vol. 265, no. 2, pp. 341350, 2003.

[25] J. L. Morales, J. A. Moreno, and F. Alhama, "Application of the network method to simulate elastostatic problems defined by potential functions. Applications to axisymmetrical hollow bodies," International Journal of Computer Mathematics, vol. 89, no. 13-14, pp. 1794-1807, 2012.
[26] J. Z. Jordán, "Network method to study the transient heat transfer problem in a vertical channel with viscous dissipation," International Communications in Heat and Mass Transfer, vol. 33, no. 9, pp. 1079-1087, 2006.

[27] PSPICE 6.0, 92718, Microsim Corporation Fairbanks, Irvine, Calif, USA, 1994. 


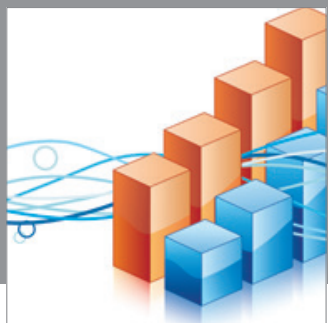

Advances in

Operations Research

mansans

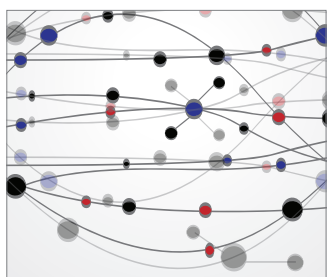

The Scientific World Journal
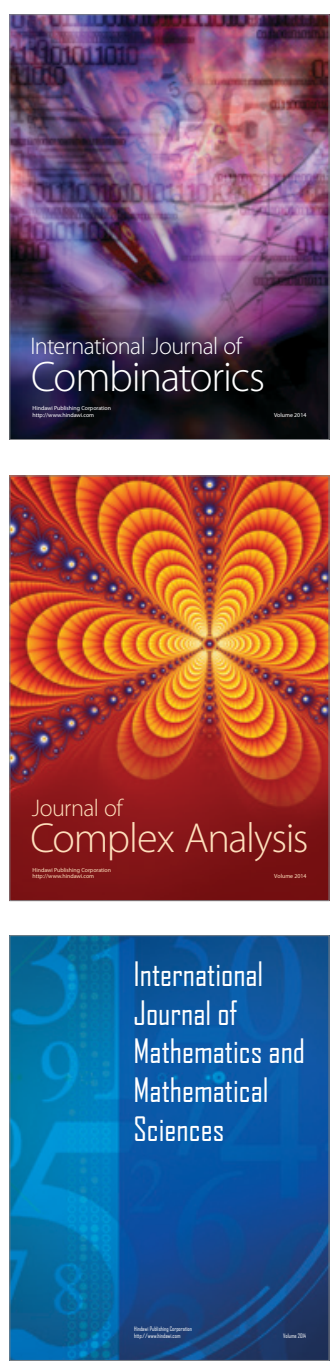
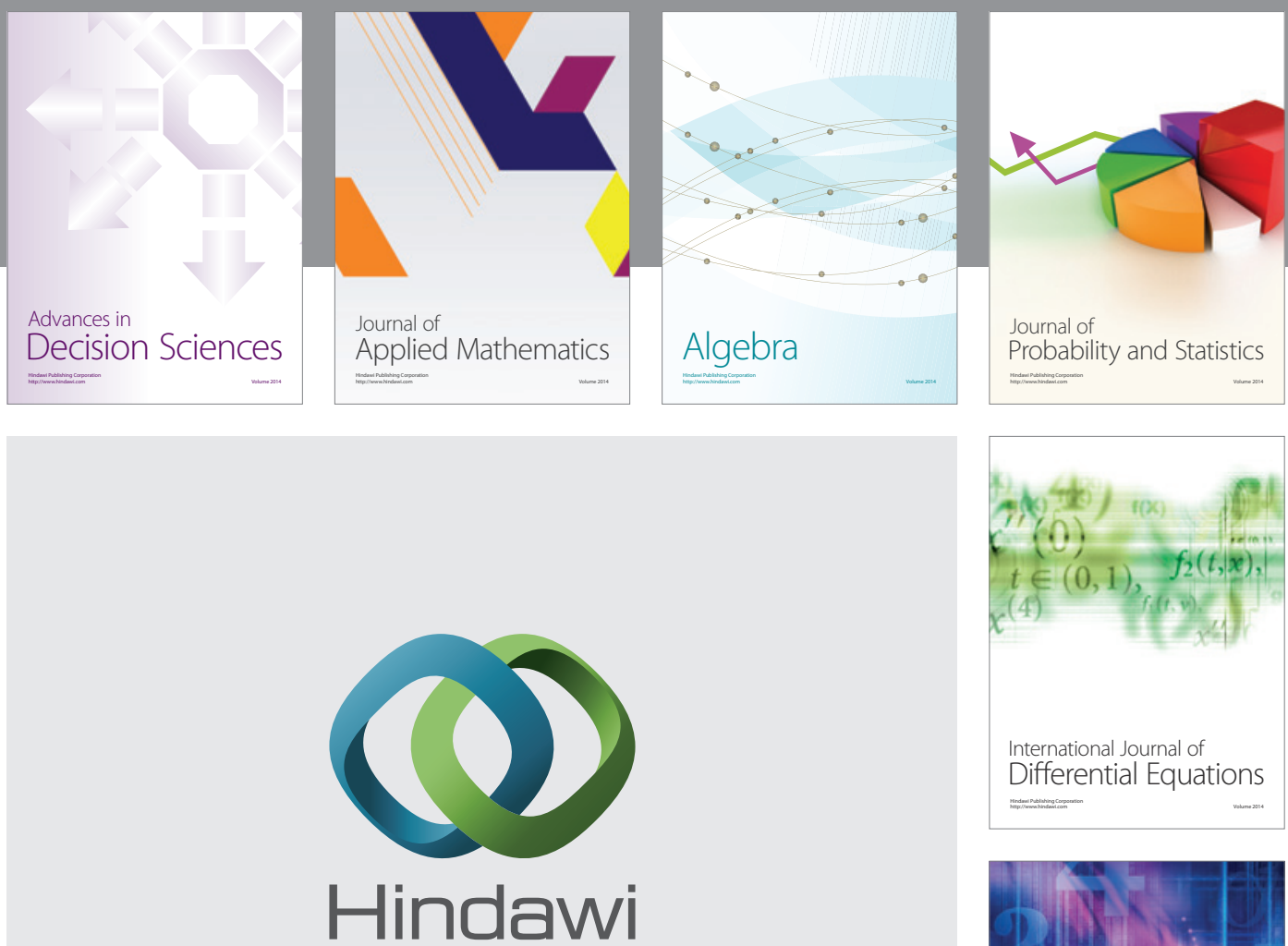

Submit your manuscripts at http://www.hindawi.com
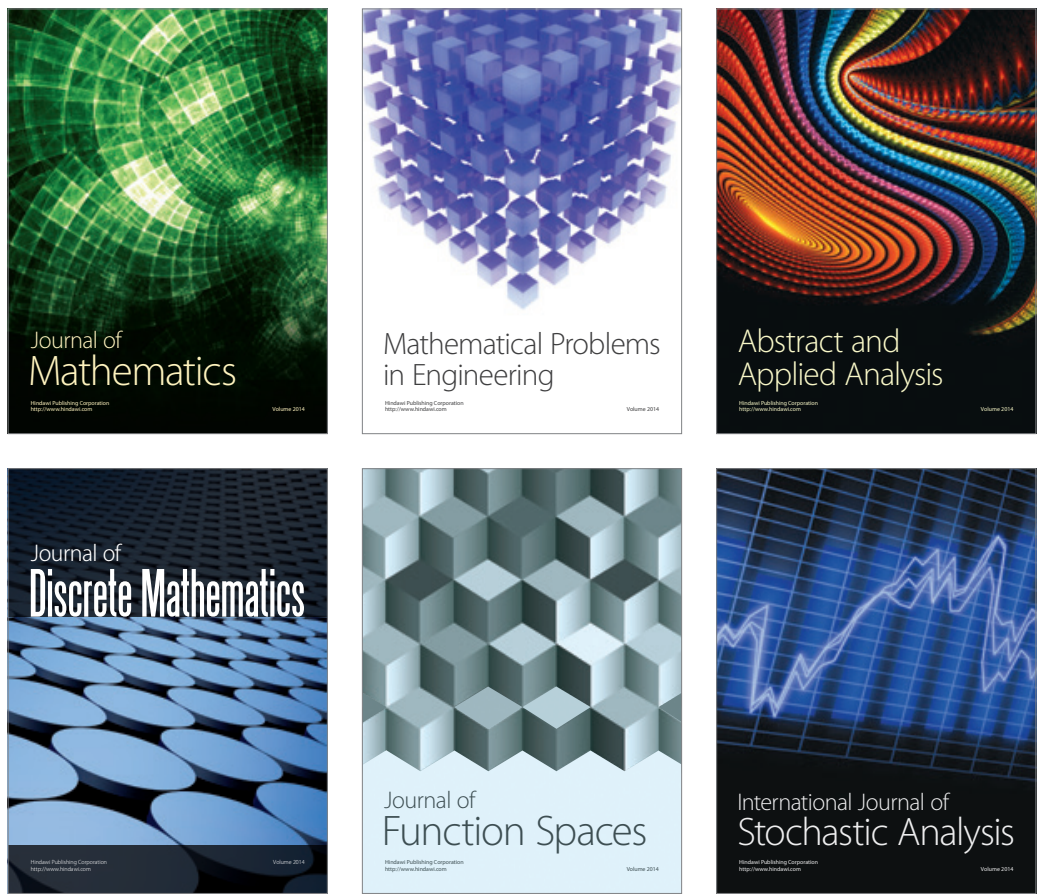

Journal of

Function Spaces

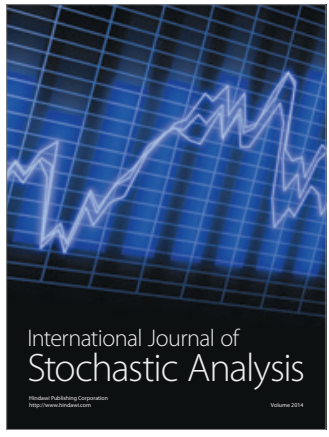

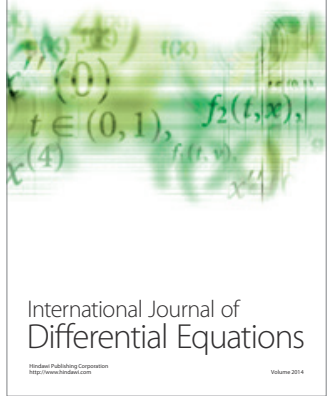
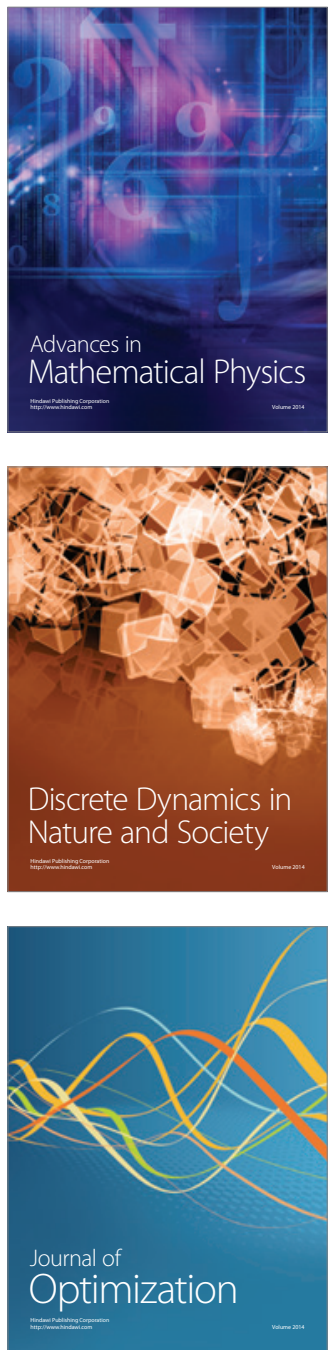